\title{
Fecal Microbiota Transplantation for Grade IV Steroid Refractory GI-GvHD: Interim Results a Non- randomized, Open-label, Phase 1 Clinical Study
}

\section{Ye Zhao}

First Affiliated Hospital of Soochow University

\section{Xuwei Li}

Soochow University

\section{YUJING ZHOU}

First Affiliated Hospital of Soochow University

\section{XIAO MA}

First Affiliated Hospital of Soochow University

\section{FENG CHEN}

First Affiliated Hospital of Soochow University

XIAOHUI HU

First Affiliated Hospital of Soochow University

\section{Song Jin}

First Affiliated Hospital of Soochow University

\section{Xuefeng He}

First Affiliated Hospital of Soochow University

Jin Gao

Soochow University

\section{Baoli Zhu}

Chinese Academy of Sciences

Depei Wu ( $\nabla$ wudepei@suda.edu.cn )

First Affiliated Hospital of Soochow University

Xiaofei Qi ( $\nabla$ qixf-sz@hotmail.com )

First Affiliated Hospital of Soochow University https://orcid.org/0000-0003-0879-7344

\section{Research}

Keywords: Fecal Microbiota Transplantation, GvHD, steroid refractory

Posted Date: July 29th, 2020

DOI: https://doi.org/10.21203/rs.3.rs-48404/v1 
License: (c) (i) This work is licensed under a Creative Commons Attribution 4.0 International License. Read Full License 


\section{Fecal Microbiota Transplantation for Grade IV Steroid Refractory GI-GvHD: Interim Results a Non-randomized, Open-label, Phase 1 Clinical Study}

Ye Zhao ${ }^{1,3,5,6^{*}}$, Xue-wei $\mathrm{Li}^{1,3,4,6^{*}}$, Yu-jing Zhou ${ }^{7^{*}}$, Xiao Ma ${ }^{1,3,5}$, Feng Chen ${ }^{1,3,5}$, Xiao-hui Hu ${ }^{1,3,5}$, Song

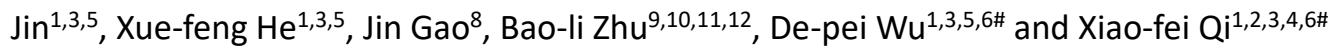

Departments of ${ }^{1}$ Hematology \& ${ }^{2}$ Urology, the First Affiliated Hospital of Soochow University, Suzhou, China; ${ }^{3}$ National Clinical Research Center for Hematologic Diseases, Jiangsu Institute of Hematology, Collaborative Innovation Center of Hematology, Suzhou, China; ${ }^{4}$ Cyrus Tang Hematology Center, Soochow university, Suzhou, China; 5 Institute of Blood and Marrow Transplantation, Soochow university, Suzhou, China; ${ }^{6}$ Key Laboratory of Thrombosis and Hemostasis of Ministry of Health, Suzhou, China; ${ }^{7}$ Center for Clinical Laboratory, the First Affiliated Hospital of Soochow University, Suzhou, China; ${ }^{8}$ State Key Laboratory of Radiation Medicine and Protection, School of Radiation Medicine and Protection, Soochow university, Suzhou, China; ${ }^{9}$ CAS Key Laboratory of Pathogenic Microbiology and Immunology, Institute of Microbiology, Chinese Academy of Sciences, Beijing, China; ${ }^{10}$ Savaid Medical School, University of Chinese Academy of Sciences, Beijing, China; ${ }^{11}$ Beijing Key Laboratory of Antimicrobial Resistance and Pathogen Genomics, Beijing, China; ${ }^{12}$ Department of Pathogenic Biology, School of Basic Medical Sciences, Southwest Medical University, Luzhou, Sichuan, China

* Y Zhao, X Li, Y Zhou contributed equally to this work.

\# Correspondence to: Prof Depei Wu, the First Affiliated Hospital of Soochow University, Suzhou, 215006, China wudepei@suda.edu.cn; Prof Xiaofei Qi, the First Affiliated Hospital of Soochow University, Suzhou, 215006, China qixf-sz@hotmail.com

\section{Runing title: FMT in GvHD}




\section{Abstract}

Background: Gastrointestinal (GI) tract graft-vs-host disease (GvHD) is a major cause of post-allo-HCT morbidity and mortality. Patients with steroid-refractory GI-GvHD face a poor prognosis and limited therapeutic options. Here, we report an interim analysis on the safety and efficacy of fecal microbiota transplantation (FMT) in treating steroid-refractory GI-GvHD.

Methods: We did a non-randomized, open-label, phase 1 clinical study on patients with grade IV steroid-refractory GI-GvHD. FMT efficacy was evaluated using indexes of abdominal pain, diarrhea and bloody purulent stool at 14 and 21 days after the diagnosis of steroid-refractory GI-GvHD. The primary outcomes referred to clinical complete remission or partial remission. Secondary outcomes referred to EFS (event free survival) and OS (overall survival) at Day 90 and the end of the research. Safety was evaluated according to adverse events during FMT and the whole follow-up period. The study was registered with ClinicalTrials.gov as \#NCT03148743.

Results: A total of 56 patients with steroid-refractory GI-GvHD were enrolled. Of them, 24 patients with grade IV steroid-refractory GI-GvHD were assigned to FMT and 18 to the control group. The characteristics of the two group patients at baseline were similar. At Day 14 after FMT, 13 (54.2\%) patients in FMT group and none (0\%) of 18 control group achieved clinical remission $(p<0.05)$, while $20(83 \%)$ patients in FMT group and $7(39 \%)$ in control group showed effective response (clinical remission+partial remission) (RR 7.86, 
$95 \% \mathrm{Cl} 1.88-32.9 ; \mathrm{p}=0.005)$. At Day 21 , the clinical remission was significantly greater in FMT group than in control group (14 (58.3\%) of 24 vs 3(16\%) of 18; RR $6.0,95 \% \mathrm{Cl} 1.22-29.45 ; p=0.027)$. Within a follow up of 90 days, the FMT group showed better OS (HR, 7.0; 95\% Cl, 1.53-32.08; $p=0.012)$. At the end of the research, the median survival time was $>600$ days in FMT group and 107 days in control group (HR, 4.73; $95 \% \mathrm{Cl}, 1.58-14.14 ; \mathrm{p}=0.005)$. Both the EFS $(\mathrm{HR}, 0.24 ; 95 \% \mathrm{Cl}, 0.06-0.95 ; \mathrm{p}=0.055)$ and $\mathrm{OS}(\mathrm{HR}, 5.97 ; 95 \% \mathrm{Cl}, 1.52-23.43$ $\mathrm{p}=0.01$ ) kept increasing during the follow-up in FMT group. Overall, the mortality rate was lower in FMT group $(H R, 5.97 ; 95 \% \mathrm{Cl}, 1.52-23.43 ; p=0.01)$. No difference was observed in the occurrence of other side effects, such as hemorrhagic cystitis, infection of bacteria \& fungi, CMV\&EBV, septicemia, TMA, cardiac events, thrombocytopenia and epilepsy.

Conclusions: The diversity of intestinal microbiota can be affected by allo-HSCT. FMT is effective and safe in treating grade IV steroid refractory GI-GvHD.

Keywords: Fecal Microbiota Transplantation, GvHD, steroid refractory 


\section{Background}

As a treatment for most acute leukemia, the hematopoietic stem cell transplantation (HSCT) can offer a potential cure but may have many complications that include infections, multi-organ failure, and graft-versus-host disease (GvHD)[1-3]. GvHD, especially gut acute GvHD (Gl-aGvHD), is a major cause of post-allo-HCT morbidity and mortality[3,4].

Conventionally, glucocorticoids are used in the first-line therapy for GI-GvHD. Unfortunately, almost half of the patients do not respond efficiently[3,4]. The survival of patients developing GI-GvHD treated with standard steroid is in a range of $5-30 \%[4-7]$. By far, few second-line treatments have been established and are urgently needed[7].

Human gut microbiota is associated with many chronic disease which is composed of more than 100 trillion microbes[8]. The influence of intestinal microbiota on immune responses, including post-allo-HCT, has been increasingly recognized[9-11], and became one of the main targets for the treatment of acute GvHD[3,7]. Under normal physiological condition, the diversity of gut microbiota in human Gl tract is associated with intestinal inflammation and immune responses[3,12] that may affect the outcome of GvHD treatment. As the intestinal microbial diversity collapses after allo-HSCT[13,14], the abnormal gut microbiota may damage Gl mucosa, and consequently influencing the immune response[3].

The fecal microbiota transplant (FMT), a procedure that use the clinical 
method of infusing a fecal suspension from a healthy donor into recipient's GI tract, can quickly restore the recipient's intestinal microbiota and repair the intestinal mucosal barrier, that may resolve the inflammatory response and readjust the immune system[15,16]. As a novel therapeutic method, FMT has been proven to be effective for recurrent clostridium difficile infection[17,18].

Our pilot study and other available research suggest that FMT can serve as a therapeutic option in treating steroid-refractory GI-GvHD[3,13,14,19]. Here, we assess the safety and efficacy of the FMT in a phase I study involving patients with steroid-refractory gastrointestinal tract GvHD.

\section{Results}

\section{Patient Characteristics}

A total of 56 patients with steroid-refractory GI-GvHD were enrolled. Clostridium difficile infection was not observed among all patients, and were not responders to methylprednisolone (mPSL) at $\geqslant 2 \mathrm{mg} / \mathrm{kg}$ per day. Immunosuppressant as the secondary-line therapy was given to all the patients. Eight patients were excluded, including four reluctant to participate in the study and four failing to meet inclusion criteria (one for primary disease recurrence, two for combined TMA, one for combined CMV before FMT). Of 27 patients in FMT group, the data of three patients with < grade IV GI-GvHD were not selected for statistical analysis. Of 21 patients in the control group, the data of three patients (one with missed follow-up and two with < grade IV 
GI-GvHD) were not used for statistical analysis (Fig.1).

The patients' information were shown in Table 1 and supplement table1. For 24 FMT patients, the median age was 29 years (range 13 to 55). The male/female ratio was $16 / 8$. The median stool volume was $865 \mathrm{~mL} /$ day (range 360 to $2,100 \mathrm{~mL} /$ day). The median stool frequency was 6 times/day (range 3 to 21 times/day). The median abdominal pain score was 3 (range 1 to 4) (Table 1 and Supplement Table1). For the 18 patients in the control group, the median age was 31.5 years old (range 13 to 59 ) (vs FMT group $p>0.05$ ). The male/female ratio was $7 / 11$ (vs FMT group $p>0.05$ ). The median stool volume was $510 \mathrm{~mL} /$ day (range 250 to $1,400 \mathrm{~mL} /$ day) (vs FMT group $p<0.05$ ). The median stool frequency was 5 times/day (range 3 to 20 times/day) (vs FMT group $p>0.05$ ). The median abdominal pain score was 2 (range 0 to 4 ) (vs FMT group $p>0.05$ ) (Table 1 and Supplement Table1). No difference was observed in the occurrence of hematologic disease, stem cells donor gender match and stem cells donor relationship in two group(Table 1 and Supplement Table1). In FMT group, 11 patients received 2 times of FMT, 9 patients received 1 time of FMT, 3 patients received 3 times of FMT and 1 patients received 6 times of FMT (Supplement Table1).

\section{Clinical Outcomes}

With Cox regression model, we first declared that immunosuppressant did not affect the outcomes of the two groups (Supplement Table1). 
At Day 14(day after steroid-refractory GI-GvHD was diagnosed), 13 (27\%) patients in FMT group and none in control group achieved clinical remission according to modified intention-to-treat analysis $(p<0.05)$. Meanwhile, $20(83 \%)$ patients in FMT group and 7(39\%) patients in control group showed effective response (clinical remission + partial remission) $(\mathrm{RR} 7.86,95 \% \mathrm{Cl} 1.88-32.9$; $\mathrm{p}=0.005)$. Three (12.5\%) patients died in FMT group and one (5.5\%) patient died in control group (RR 0.41, 95\% $\mathrm{Cl}$ 0.04-4.33; $\mathrm{p}=0.46$ )(Fig.2)(Table 2 and Supplement Table1). No relapse of GI-GvHD was recorded in all patients at this time point.

At Day 21, clinical assessments showed that clinical remission was significantly more obvious in FMT group than in control group (14[58.3\%] of 24 vs $3[16 \%]$ of 18 ; RR $6.0,95 \% \mathrm{Cl} 1.22-29.45 ; \mathrm{p}=0.027)$, but clinical response did not difference (16 [66.7\%] of 24 vs 9 [50\%] of 18 ; RR $4.0,95 \% \mathrm{Cl}$ 0.84-19.16; $p=0.083)$. Three patients died in FMT group and two patients died in control group (3 [12.5\%] of 24 vs 2 [11\%] of 18 ; RR $0.48,95 \% \mathrm{Cl} 0.08-2.79$; $\mathrm{p}=0.41$ ). Two patients in FMT group and two patients in control group showed disease relapse, but no significant difference (2 [8.3\%] of 24 vs 2 [11\%] of 18 ; RR 0.73, 95\%Cl 0.09-5.72; p=0.76). (Fig.2) (Table 2 and Supplement Table1). At $28^{\text {th }}$ day, the rate of clinical remission and effective response were higher in FMT group than control group(Supplement Table2).

Within 90 days of follow-up, EFS showed no significant difference between two groups (HR 0.36, 95\% $\mathrm{Cl}, 0.1-1.28 ; \mathrm{p}=0.11$ ) (Fig.3A). The FMT 
group showed better OS (HR 7.0, 95\%Cl, 1.53-32.08; $p=0.012$ ) (Fig.3B). At the end of the research, the median survival time was $>600$ days in FMT group and 107 days in control group ( $\mathrm{HR} 4.73,95 \% \mathrm{Cl}, 1.58-14.14 ; \mathrm{p}=0.005)$. Both the EFS (HR 0.24, 95\% Cl, 0.06-0.95; $\mathrm{p}=0.055)$ and OS $(\mathrm{HR} 5.97,95 \% \mathrm{Cl}$, 1.52-23.43; $p=0.01$ ) kept increasing during the follow-up time in FMT group(Fig.3CD).

\section{Safety of FMT}

Only one patient experienced thrombocytopenia after FMT and one patient developed cardiac event at day 3 after FMT. Although we were not sure whether there was a possible association between these events and FMT, it cannot be completely exclude. No other severe adverse events were observed in FMT group during seven days of follow-up after FMT. Other common adverse events include incomplete ileus in one patient, fever in one patient, vomiting and low fever in two patients, and grade-3 rash in two patients, no special treatment only symptomatic treatment need for these patients.

In overall results, the mortality rate was low in FMT group (HR 5.97, $95 \% \mathrm{Cl}, 1.52-23.43 ; \mathrm{p}=0.01)$. No significant difference was observed between two group in the occurrence of hemorrhagic cystitis $(p>0.05)$, bacteria \& fungi infection ( $p>0.05)$, CMV\&EBV infection $(p>0.05)$, septicemia $(p>0.05)$, TMA $(p>0.05)$, cardiac events $(p>0.05)$, thrombocytopenia $(p>0.05)$ and epilepsy ( $p>0.05)$. (Table 3 and Supplement Table1) 


\section{Fecal Microbiota and Immunity functions Analysis}

Given the severity and emergency of steroid-refractory grade IV GI-GvHD, only 10 patients got fecal samples at baseline and weeks 1 after FMT. Available fecal samples were used for microbiota analyses $(\mathrm{N}=10)$. Compared with that of the donors, the diversity of fecal microbiota in fecal samples of the patients was decreased (Fig.4A). Proteobacteria increased while firmicutes decreased at phylum level in the microbiota of the patients(Fig.4B). After Week 1, the microbiota composition was reconstructed in FMT patients, showing a trend back to normal(Fig.4CD). The bacterial diversity improved at Week 1 after FMT in half patients (5/10) (Supplement Table1). Similar to the results of our prior study, the ratio of firmicutes to proteobacteria was restored $(7 / 10)$, proteobacteria decreased(9/10), and firmicutes increased (6/10) after FMT(Figure S1). Bacteroidetes increased (7/10) in the fecal microbiota of patients with steroid-refractory GI-GvHD (Supplement Table1) after FMT.

\section{Discussion}

Gut-GvHD related complications, especially steroid-refractory GI-GvHD, appeared to be one of cause of post-transplantation death[3,4], and the disruption of gut microbiota was linked to GvHD and transplant-related mortality[20,21].

The FMT may re-structured the gut microbiota of a patient which may 
consequently reinforce the patient's immune system[18]. The FMT had been proven to be very effective for the treatment of recurrent Clostridium difficile infection[17] and other human diseases (inflammatory bowel disease) $[3,15,16]$. Some case reports and our pilot study suggested that FMT may serve as a therapeutic option for steroid-refractory GI-GvHD[3,13,14,19].

In this research, we enrolled 56 patients with steroid-refractory gastrointestinal tract GvHD. Twenty four patients with grade-IV steroid-refractory GI-GvHD were given FMT(Fig1). As a result, the microbial richness in diversity and abundance were increased after FMT in most patients in comparison with the patient's gut microbiota before treatment. The microbiota composition was also restored. Beneficial bacteria, such as bacteroidetes or firmicutes, became dominant after FMT in most patients(Fig4).

In this research, the diarrhea and abdominal pain were attenuated after FMT(Fig2). The proportion of patients with clinical remission and effective response was higher at 2 or 3 weeks after FMT(Table 2). During a follow-up of 90 days, although EFS showed no significant difference between the two groups, the FMT group still showed better OS. In overall results, the median survival was longer in FMT group than that in control group. Furthermore, both EFS and OS kept increasing during the follow-up in FMT group(Fig3).

In our research, only one case of thrombocytopenia, one case of cardiac event, and no cases of other severe adverse events were observed in FMT 
group during 7 days' follow-up after FMT. In overall results, FMT did not increase the probability of bacteria \& fungi infection, CMV\&EBV infection and septicemia. The incidences of hemorrhagic cystitis, TMA, cardiac events, thrombocytopenia and epilepsy in two groups were similar(Table 3). Some papers reported that FMT transmitted drug-resistant $E$. coli, leading to patient death[22]. No similar events were observed in our study, which may be attributed to our strict FMT criteria.

\section{Conclusion}

Although up to date no reports from any phase I clinical trial of FMT treated GI GvHD were found, our study was also limited in some aspects. It was conducted at a single institution, and thus, our findings may not be applied directly to patients at other institutions[20]. Another, given the severity and emergency of steroid-refractory grade IV GI-GvHD, the trial was not randomized and double blind controlled. And also for this, only partial patients got all samples for gut microbiome analysis, we couldn't get the gut microbiome dynamics in all patients, and immunosuppressant was given and its effect could not be completely ruled out in this research. Next, not all patients showed similar responses to FMT, and we did not get enough data to compare responding and non-responding patients, which may be explained by the pathological complexity of steroid-refractory GI-GvHD.

In summary, FMT may be effective and safe in treating GI-GvHD, which 
should be verified with more studies.

\section{Methods}

\section{Study design and participants}

We undertook an open-label, non-randomized, phase 1 clinical study at the First Affiliated Hospital of Soochow University. Protocols and other trial related procedures were approved by the Institutional Review Board of the Hospital. All the patients signed written informed consent. FMT group and control group (Without FMT) were set according the patients decision after introducing the possible benefit and disadvantages of fecal bacteria transplantation. FMT was performed after steroid-refractory GI-GvHD was diagnosed. All the patients were given second-line immunosuppressant treatment. Only the FMT group received FMT. The study was registered with ClinicalTrials.gov as \#NCT03148743. The Center for International Blood and Marrow Transplant Research (CIBMTR) criteria were used to assess the grades of Gl GvHD[3,23,24]. Criteria for diagnosing steroid-refractory gut GvHD had been described previously[3]. We excluded the patients with uncontrollable infection, irreversible organ failure, and other abnormal conditions that might interfere with the evaluation(Supplement Protocol).

\section{Procedures}

The fecal materials were handled in sealed, fully automatic machines 
GenFMTer (Nanjing, China). The fecal microbiota collected from four healthy donors (two females aged 23 years, and two males aged 20 years) were conserved in $-80^{\circ} \mathrm{C}$ with glycerine(Supplement protocol). As these patients couldn't tolerate gastroscopy or enteroscopy, forty to fifty $\mathrm{ml}$ of frozen fecal microbiota were suspended in $150-200 \mathrm{ml}$ of warm normal saline and delivered into the intestine of the recipients through a nasojejunal tube or gastric tube after steroid-refractory GI-GvHD was diagnosed[3]. If not got improvement, FMT would be repeated in the following week.

\section{Outcomes}

The primary outcomes were described with clinical remission or partial remission at Day 14th, Day 21th and Day 28th after steroid-refractory GI-GvHD was diagnosed.

Secondary outcomes were: EFS and OS at Day 90 after steroid-refractory GI-GvHD was diagnosed; EFS and OS after steroid-refractory GI-GvHD was recorded till the end of November1 2018

FMT efficacy were evaluated according to the severity of symptoms such as abdominal pain, diarrhea (frequency and volume), and bloody purulent stool within 14 and 21 days after FMT was accomplished. For abdominal pain score, 0.5 was given to occasional pain, 1 to mild pain, 2 to moderate pain, 3 to severe pain without intervention, and 4 to severe pain with intervention. Clinical remission was defined as a condition in which diarrhea and intestinal 
spasms and/or bleeding disappeared, or stool volume decreased by $\geqslant 500 \mathrm{~mL}$ on average within 3 days. Clinical improvement was defined as a condition in which the stool volume decreased by $<500 \mathrm{~mL}$, or the abdominal pain value and bleeding relieved. The period during follow-up after first FMT with no progress of GI-GvHD, no death, no GvHD involvement in other organs, no new infection with CMV and EBV were defined as event free survival time (EFS) [3]. OS (overall survival) referred to the period from when steroid-refractory GI-GvHD was diagnosed to November1 2018. All deaths, including relapse related or other causes in these period, were included in the statistics.

For each patient, the safety was evaluated according to adverse events (including death or drop-out) during FMT and follow-up time.

\section{Stool Sample Collection and Microbial Community Analysis}

Fecal samples were stored at $-80^{\circ} \mathrm{C}$ until DNA extraction. After DNA was extracted, we successfully detected bacterial $16 \mathrm{~S}$ rDNA in all samples with polymerase chain reaction (PCR) using general bacterial primers (16S V4-V5): 515F:5'-GTGCCAGCMGCCGCGGTAA-3'; 926R: 5'-CCGTCAATTCMTTTGA -GTTT-3'. After purified, the pooling libraries were sequenced by $2 * 300 \mathrm{bp}$ paired-end sequencing on the MiSeq platform (Tiny Gene, Shanghai) using MiSeq v3 Reagent Kit (Illumina). We used a combination of Mothur, UPARSE and $\mathrm{R}$ software to analyze the $16 \mathrm{~S}$ sequences data. Composition of fecal bacteria was analyzed at phylum level. Moreover, the Shannon diversity index 
was used to depict the diversity of microbiota(Supplement Methods) [3,25].

\section{Statistical Analysis}

SPSS16.0 (SPSS, Inc., Chicago, IL, USA) for statistical analyses was used to construct actuarial rate curves and to calculate log-rank hazard ratios (HRs) and significance determinations, Fisher's exact tests, and risk determinations. Cochran's and mantel-haenszel statistical methods examined the differences between the groups. The survival package from "R" statistical software (Vienna, Austria) was used for permutation tests. For the latter, variables were determined for each day of the patient's hospitalization and HRs, confidence intervals, and significance determinations were calculated using Cox proportional hazards models with time varying covariates.

\section{Declarations}

\section{Ethics approval and consent to participate}

Protocols and other trial related procedures were approved by the Institutional Review Board of the Hospital. All the patients signed written informed consent.

\section{Consent for publication}

No applicable 
Please contact author for data requests

\section{Competing interests}

The authors declare that they have no competing interests.

\section{Funding}

This study was supported in part by grants from of National Key R\&D Program of China (2017YFA0104502,2016YFC0902800); the National Nature Science Foundation of China (81170468 and 81470346); Foundation of National Clinical Research Center for Hematologic Disease of China (2020ZKPC01); the Priority Academic Program Development of Jiangsu Higher Education Institutions(PAPD).

\section{Authors' contributions}

$\mathrm{XQ}, \mathrm{BZ}$ and DW contributed to the study concept and design. $\mathrm{YZ}, \mathrm{XL}, \mathrm{XW}, \mathrm{FC}$, SJ, $\mathrm{XH}$ and $\mathrm{XM}$ collected the clinical samples. $\mathrm{XL}$ and $\mathrm{YZ}$ performed the experiments. $X Q$, JG, and $Y Z$ performed bioinformatics analyses. $X Q, Y Z$ and BZ wrote the manuscript. XQ, BZ and DW supervised the study. All authors read and approved the final version of the report.

\section{Acknowledgments}

We are grateful to Dr. Fanming Zhang and Pan Li for expert technical 
assistance. 


\section{References}

1. Holtan SG , Pasquini M , Weisdorf DJ. Acute GVHD: a bench to bedside update. Blood. 2014; 124: 363-373.

2. Copelan EA. Hematopoietic stem-cell transplantation. N Engl J Med. 2006; 354:1813-26.

3. Qi X, Li X, Zhao Y, Wu X, Chen F, Ma X, Zhang F, Wu D. Treating Steroid Refractory Intestinal Acute Graft-vs.-Host Disease With Fecal Microbiota Transplantation: A Pilot Study. Front Immunol. 2018; 9:2195.

4. McDonald GB. How I treat acute graft-versus-host disease of the gastrointestinal tract and the liver. Blood. 2016; 127:1544-50.

5. Martin PJ, Rizzo JD, Wingard JR, Ballen K, Curtin PT, Cutler C, Litzow MR, Nieto Y, Savani BN, Schriber JR, Shaughnessy PJ, Wall DA, Carpenter PA. First- and second-line systemic treatment of acute graft versus host disease: recommendations of the american society of blood and marrow transplantation. Biol Blood Marrow Transplant. 2012; 18:1150-63.

6. Castilla-Llorente C, Martin PJ, McDonald GB, Storer BE, Appelbaum FR, Deeg HJ, Mielcarek M, Shulman H, Storb R, Nash RA. Prognostic factors and outcomes of severe gastrointestinal GVHD after allogeneic hematopoietic cell transplantation. Bone Marrow Transplant. 2014; 49:966-71.

7. Köhler N, Zeiser R. Intestinal microbiota influence immune tolerance post allogeneic hematopoietic cell transplantation and Intestinal GVHD. Front Immunol. 2019; 9:3179.

8. Turnbaugh PJ, Ley RE, Hamady M, Fraser-Liggett CM, Knight R, Gordon Jl. The human microbiome project. Nature. 2007; 449: 804-10.

9. Jenq RR, Ubeda C, Taur Y, Menezes CC, Khanin R, Dudakov JA, Liu C, West ML, Singer NV, Equinda MJ, Gobourne A, Lipuma L, Young LF, Smith OM, Ghosh A, Hanash AM, Goldberg JD, Aoyama K, Blazar BR, Pamer EG, van den Brink MR. Regulation of intestinal inflammation by microbiota following allogeneic bone marrow transplantation. J Exp Med. 2012;209:903-11.

10. Round JL, Mazmanian SK. The gut microbiota shapes intestinal immune responses during health and disease. Nat Rev Immunol. 2009; 9:313-23.

11. Penack O, Holler E, van den Brink MR. Graft-versus-host disease: regulation by microbe-associated molecules and innate immune receptors. Blood. 2010;115:1865-172.

12. Buffie CG, Pamer EG. Microbiota-mediated colonization resistance against intestinal pathogens. Nat Rev Immunol. 2013; 13:790-801.

13. Spindelboeck W, Schulz E, Uhl B, Kashofer K, Aigelsreiter A, Zinke-Cerwenka W, Mulabecirovic A, Kump PK, Halwachs B, Gorkiewicz G, Sill H, Greinix H, Högenauer C, Neumeister P. Repeated fecal microbiota transplantations attenuate diarrhea and lead to sustained changes in the fecal microbiota in acute, refractory gastrointestinal graft-versus-host-disease. Haematologica. 2017; 102:210-3.

14. Kakihana K, Fujioka Y, Suda W, Najima Y, Kuwata G, Sasajima S, Mimura I, Morita H, Sugiyama D, Nishikawa H, Hattori M, Hino Y, Ikegawa S, Yamamoto K, Toya T, Doki N, Koizumi K, Honda K, Ohashi K Fecal microbiota transplantation for patients with steroid-resistant acute graft-versus-host disease of the gut. Blood. 2016; 128:2083-8

15. Kelly CR, Kahn S, Kashyap P, Laine L, Rubin D, Atreja A, Moore T, Wu G. Update on Fecal Microbiota Transplantation 2015: Indications, Methodologies, Mechanisms, and Outlook. Gastroenterology. 2015; 149: 223-37. 
16. Weingarden AR, Vaughn BP. Intestinal microbiota, fecal microbiota transplantation, and inflammatory bowel disease. Gut Microbes. 2017; 8: 238-52.

17. Kelly CR, Ihunnah C, Fischer M, Khoruts A, Surawicz C, Afzali A, Aroniadis O, Barto A, Borody T, Giovanelli A, Gordon S, Gluck M, Hohmann EL, Kao D, Kao JY, McQuillen DP, Mellow M, Rank KM, Rao K, Ray A, Schwartz MA, Singh N, Stollman N, Suskind DL, Vindigni SM, Youngster I, Brandt L. Fecal microbiota transplant for treatment of Clostridium difficile infection in immunocompromised patients. Am J Gastroenterol. 2014; 109: 1065-71.

18. van Nood E, Vrieze A, Nieuwdorp M, Fuentes S, Zoetendal EG, de Vos WM, Visser CE, Kuijper EJ, Bartelsman JF, Tijssen JG, Speelman P, Dijkgraaf MG, Keller JJ. Duodenal infusion of donor feces for recurrent Clostridium difficile. N Engl J Med. 2013; 368:407-15.

19. Wardill HR, Secombe KR, Bryant RV, Hazenberg MD, Costello SP. Adjunctive fecal microbiota transplantation in supportive oncology: Emerging indications and considerations in immunocompromised patients. EBioMedicine. 2019; 44:730-40.

20. Taur Y, Coyte K, Schluter J, Robilotti E, Figueroa C, Gjonbalaj M, Littmann ER, Ling L, Miller L, Gyaltshen $Y$, Fontana E, Morjaria S, Gyurkocza B, Perales MA, Castro-Malaspina H, Tamari R, Ponce D, Koehne G, Barker J, Jakubowski A, Papadopoulos E, Dahi P, Sauter C, Shaffer B, Young JW, Peled J, Meagher RC, Jenq RR, van den Brink MRM, Giralt SA, Pamer EG, Xavier JB. Reconstitution of the gut microbiota of antibiotic-treated patients by autologous fecal microbiota transplant. Sci Transl Med. 2018; 10:460

21. Weber D, Oefner PJ, Hiergeist A, Koestler J, Gessner A, Weber M, Hahn J, Wolff D, Stämmler F, Spang R, Herr W, Dettmer K, Holler E. Low urinary indoxyl sulfate levels early after transplantation reflect a disrupted microbiome and are associated with poor outcome. Blood. 2015; $126: 1723-28$

22. Defilipp Z, Bloom PP, Torres Soto M, Mansour MK, Sater MRA, Huntley MH, Turbett S, Chung RT, Chen YB, Hohmann EL. Drug-Resistant E. coli Bacteremia Transmitted by Fecal Microbiota Transplant. N Engl J Med. 2019; 381:2043-50.

23. Rowlings PA, Przepiorka D, Klein JP, Gale RP, Passweg JR, Henslee-Downey PJ, Cahn JY, Calderwood S, Gratwohl A, Socié G, Abecasis MM, Sobocinski KA, Zhang MJ, Horowitz MM. Severity Index for grading acute graft-versus-host disease: retrospective comparison with Glucksberg grade. Br J Haemato.I 1997; 97:855-64.

24. Przepiorka D, Weisdorf D, Martin P, Klingemann HG, Beatty P, Hows J, Thomas ED. 1994 consensus conference on acute GVHD grading. Bone Marrow Transplant. 1995; 15:825-28.

25. Cui B, Li P, Xu L, Zhao Y, Wang H, Peng Z, Xu H, Xiang J, He Z, Zhang T, Nie Y, Wu K, Fan D, Ji G, Zhang F. Step-up fecal microbiota transplantation strategy: a pilot study for steroid-dependent ulcerative colitis. J Transl Med. 2015; 13:298-309. 


\section{Figure Legends}

Figure 1. Experimental flow diagram.

Figure 2. Clinical response to FMT. (A) Stool volumes of all patients at baseline, Day 14 and Day 21 after steroid-refractory GI-GvHD was diagnosed. (B) Stool frequency of all patients at baseline, Day 14 and Day 21 after steroid-refractory GI-GvHD was diagnosed. (C) Abdominal pain score of all patients at baseline, Day 14 and Day 21 after steroid-refractory GI-GvHD was diagnosed.

Figure 3. Kaplan-Meier curves demonstrating survival outcomes. EFS (A) and OS (B) of all patients within 90 days of follow-up time; EFS(C) and OS (D) at the end of research

\section{Figure 4. FMT improves gut microbiota diversity and composition in patients.}

(A) The diversity of fecal microbiota in all sample (Shannon's diversity index $)\left(\mathrm{n}_{\text {donor }}=4\right.$, $\left.n_{\text {patient }}=10\right)$.(B) proteobacteria and firmicutes change in donor group, pre-FMT samples. ${ }^{*} p<0.05$. (C) OTUs change in donor group, pre-FMT(OD) and post-FMT(7D) samples. (D) Analysis of fecal microbiota composition in all samples at the phylum level $\left(\mathrm{n}_{\text {donor }}=4\right.$, $\left.\mathrm{n}_{\text {patient }}=10\right)$. Each row represents a study subject. Px means patient number, Dx means donor number, xD means day after FMT. 


\section{Supplement Figure Legends}

Figure S1. Analysis of fecal microbiota in donor and 10 patients. (A) proteobacteria and firmicutes change in donor group change in donor group, pre-FMT(0D) and post-FMT(7D) samples. ${ }^{*} p<0.05$. 
Table 1 Baseline characteristics of patients

\begin{tabular}{|c|c|c|c|}
\hline & FMT & control & $\mathrm{p}$ \\
\hline median Age(min-max) & $29(13-55)$ & $31.5(13-59)$ & $>0.05$ \\
\hline Gender & & & $>0.05$ \\
\hline male & 16 & 7 & \\
\hline female & 8 & 11 & \\
\hline Volume ml & $865(360-2100)$ & $510(250-1400)$ & $<0.05^{*}$ \\
\hline \multicolumn{4}{|l|}{ median(min-max) } \\
\hline Frequencies & $6(3-21)$ & $5(3-20)$ & $>0.05$ \\
\hline Abdominal pain & $3(1-4)$ & $2(0-4)$ & $>0.05$ \\
\hline \multicolumn{4}{|l|}{ Hematologic Disease } \\
\hline AML & 8 & 9 & $>0.05$ \\
\hline ALL & 4 & 2 & $>0.05$ \\
\hline MDS & 5 & 4 & $>0.05$ \\
\hline AA & 4 & 1 & $>0.05$ \\
\hline CML & 2 & 0 & $>0.05$ \\
\hline Others & 1 & 2 & $>0.05$ \\
\hline Stem cells donor gender match & 13 & 7 & $>0.05$ \\
\hline \multicolumn{4}{|l|}{ Stem cells donor relationship } \\
\hline Haplo-HSCT & 20 & 14 & $>0.05$ \\
\hline SIB-HSCT & 2 & 3 & $>0.05$ \\
\hline URD-HSCT & 2 & 1 & $>0.05$ \\
\hline
\end{tabular}


Table 2 Clinical results of $14^{\text {th }}$ day and $21^{\text {th }}$ day

\begin{tabular}{|c|c|c|c|c|}
\hline & & $\begin{array}{c}\text { FMT } \\
(n=24)\end{array}$ & $\begin{array}{l}\text { Control } \\
(n=18)\end{array}$ & $p$ \\
\hline \multirow{3}{*}{0 day } & $\begin{array}{l}\text { Stool volume } \mathrm{ml} \\
\text { median(min-max) }\end{array}$ & $865(360-2100)$ & $510(250-1400)$ & $<0.05^{*}$ \\
\hline & Stool frequencies & $6(3-21)$ & $5(3-20)$ & $>0.05$ \\
\hline & Abdominal pain score & $3(1-4)$ & $2(0-4)$ & $>0.05$ \\
\hline \multirow{6}{*}{$14^{\text {th }}$ day } & $\begin{array}{l}\text { Stool volume } \mathrm{ml} \\
\text { median(min-max) }\end{array}$ & $200(0-1300)$ & $500(0-1700)$ & $<0.05^{*}$ \\
\hline & Stool frequencies & $2(0-9)$ & $5(0-12)$ & $<0.05^{*}$ \\
\hline & Abdominal pain score & $0(0-3)$ & $2(0-4)$ & $<0.05^{*}$ \\
\hline & $\mathrm{CR}$ & $13(54.2 \%)$ & $0(0 \%)$ & $<0.05^{*}$ \\
\hline & Efficiency(CR+PR) & $20(83.3 \%)$ & $7(39 \%)$ & $<0.05^{*}$ \\
\hline & Die & $3(12.5 \%)$ & $1(5.5 \%)$ & $>0.05$ \\
\hline \multirow{7}{*}{$21^{\text {th }}$ day } & $\begin{array}{l}\text { Stool volume ml } \\
\text { median(min-max) }\end{array}$ & $180(0-2365)$ & $450(0-1400)$ & $>0.05$ \\
\hline & Stool frequencies & $2(0-8)$ & $4(0-15)$ & $>0.05$ \\
\hline & Abdominal pain score & $0(0-3)$ & $2(0-4)$ & $>0.05$ \\
\hline & $\mathrm{CR}$ & $16(66.7 \%)$ & $3(16 \%)$ & $<0.05^{*}$ \\
\hline & Efficiency(CR+PR) & $18(75 \%)$ & $9(50 \%)$ & $>0.05$ \\
\hline & Die & $3(12.5 \%)$ & $2(11 \%)$ & $>0.05$ \\
\hline & GI-GvHD Relapse & $2(8.3 \%)$ & $2(11 \%)$ & $>0.05$ \\
\hline
\end{tabular}

$\mathrm{CR}$ (clinical remission); $\mathrm{PR}$ (partial remission) 
Table 3 Adverse events during overall follow-up time

\begin{tabular}{|c|c|c|c|c|c|c|c|c|c|}
\hline & $\begin{array}{c}\text { Hemorrhagic } \\
\text { cystitis }\end{array}$ & $\begin{array}{c}\text { CMV \& } \\
\text { EBV }\end{array}$ & TMA & $\begin{array}{l}\text { Infection rate } \\
\text { (Bacteria \& } \\
\text { fungi) }\end{array}$ & Septicemia & $\begin{array}{c}\text { Cardiac } \\
\text { event }\end{array}$ & $\begin{array}{c}\text { Thrombocytopenia } \\
\text { \& cerebral } \\
\text { hemorrhage }\end{array}$ & Epilepsy & Die \\
\hline $\begin{array}{l}\text { FMT group } \\
\qquad(n=24)\end{array}$ & $1 / 24$ & $8 / 24$ & $6 / 24$ & $5 / 24$ & $2 / 24$ & $2 / 24$ & $1 / 24$ & $1 / 24$ & $5 / 24(20.8 \%)$ \\
\hline $\begin{array}{l}\text { Control } \\
\text { group } \\
(n=18)\end{array}$ & $3 / 18$ & $4 / 18$ & $6 / 18$ & $7 / 18$ & $4 / 18$ & $1 / 18$ & $0 / 18$ & $1 / 18$ & $10 / 18(55.6 \%)$ \\
\hline $\mathrm{p}$ & $>0.05$ & $>0.05$ & $>0.05$ & $>0.05$ & $>0.05$ & $>0.05$ & $>0.05$ & $>0.05$ & $<0.05^{*}$ \\
\hline
\end{tabular}


Figures

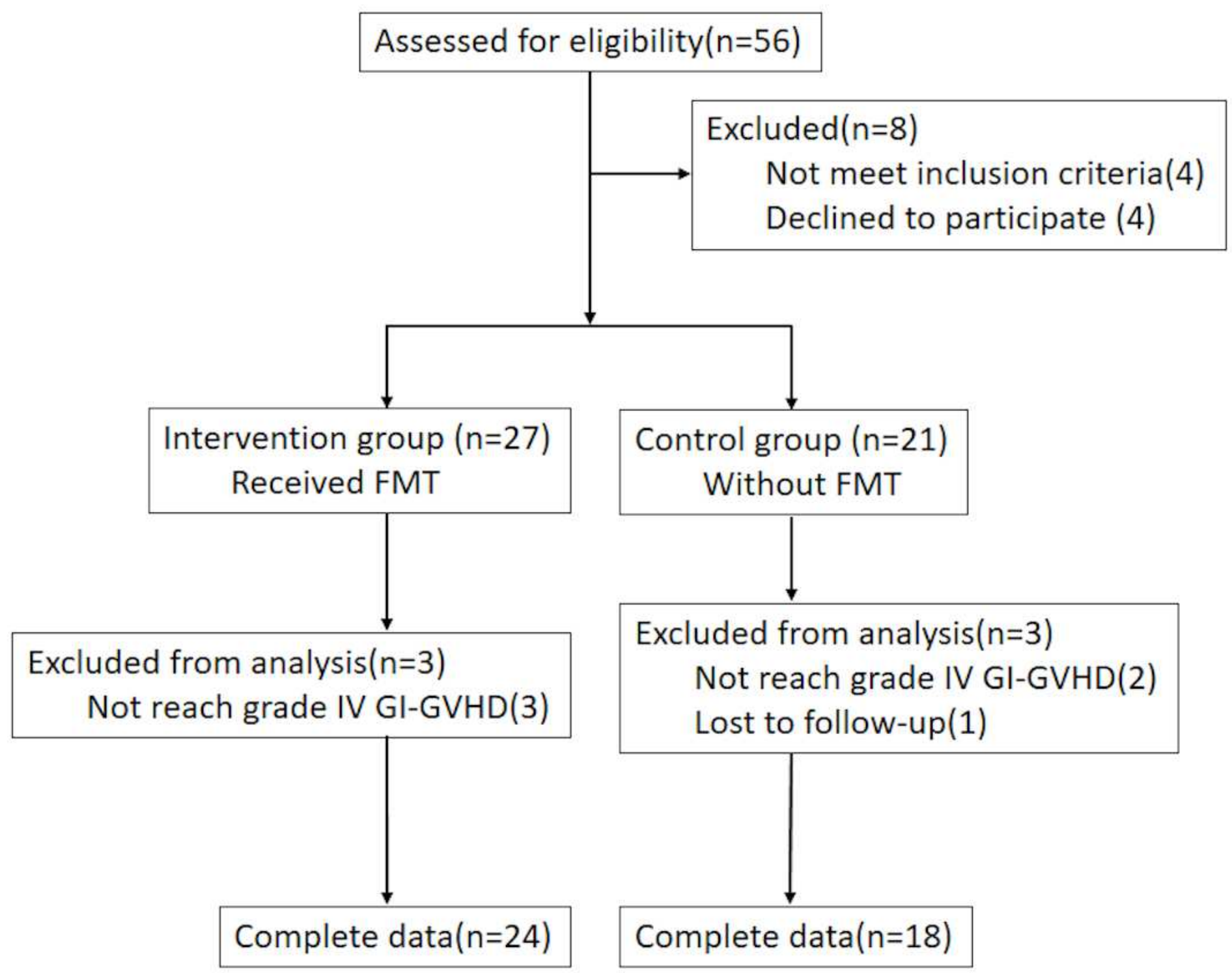

Figure 1

Experimental flow diagram 
A

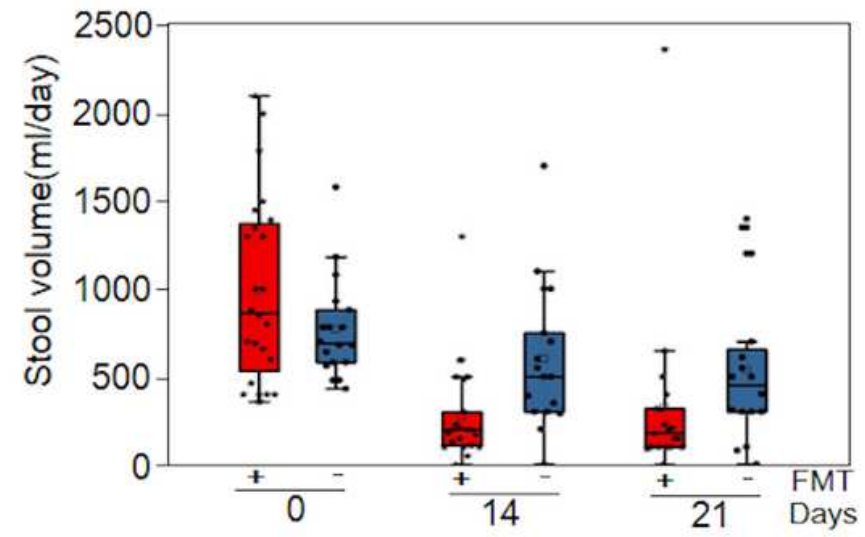

B

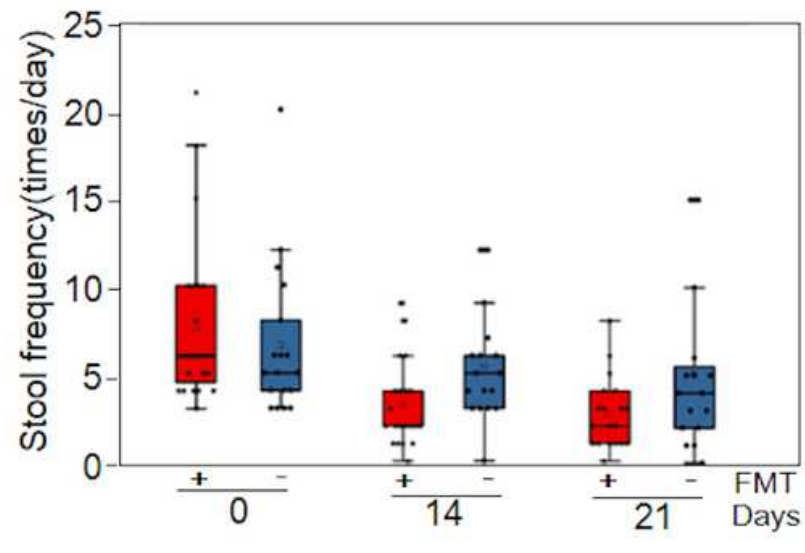

C

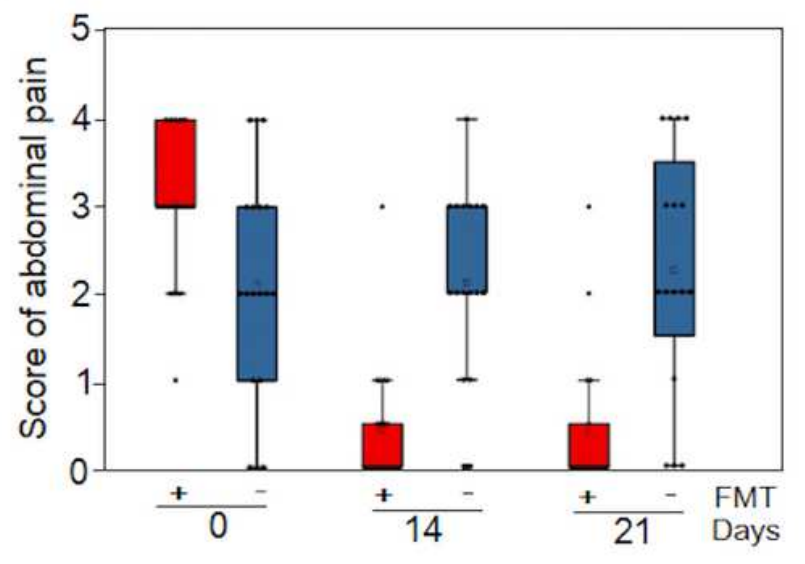

Figure 2

Clinical response to FMT. (A) Stool volumes of all patients at baseline, Day 14 and Day 21 after steroid refractory GI GvHD was diagnosed (B) Stool frequency of all patients at baseline, Day 14 and Day 21 after steroid refractory GI-GvHD was diagnosed (C) Abdominal pain score of all patients at baseline, Day 14 and Day 21 after steroid refractory GI-GvHD was diagnosed 
A

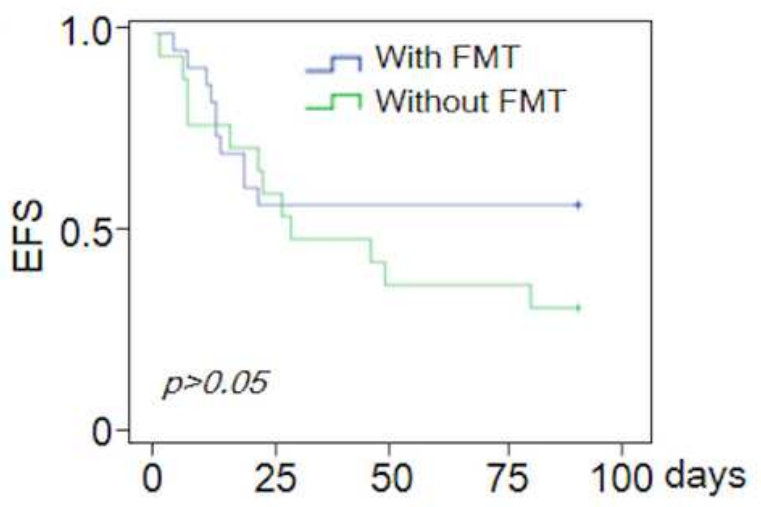

Number at Risk

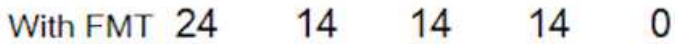
Without FMT $18 \quad 11 \quad 7 \quad 7 \quad 0$

C

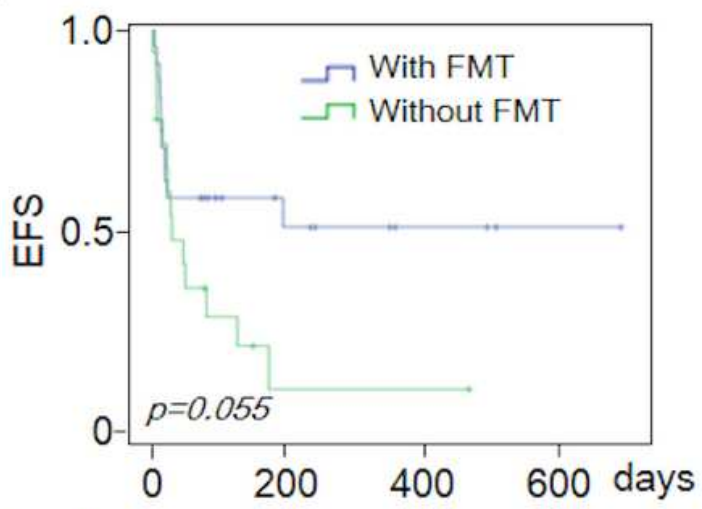

Number at Risk

$\begin{array}{lllll}\text { With FMT } & 24 & 7 & 3 & 1\end{array}$

$\begin{array}{llll}\text { Without FMT } 18 & 1 & 1 & 0\end{array}$
B

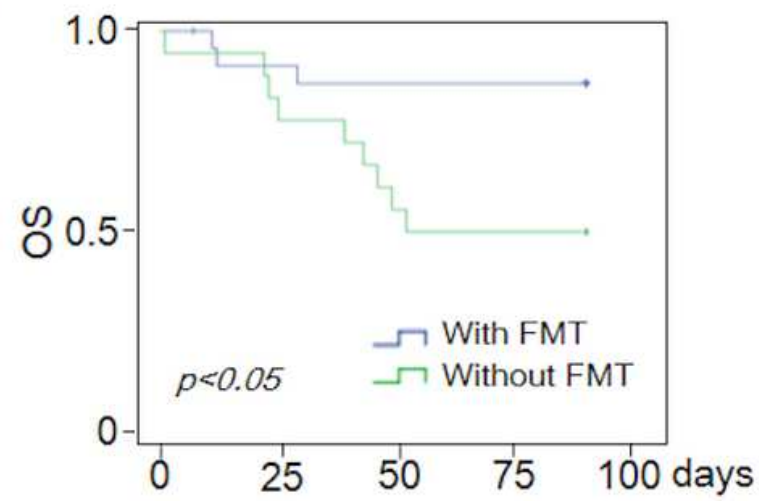

Number at Risk

\begin{tabular}{|c|c|c|c|}
\hline With FMT 24 & 21 & 20 & \\
\hline thout FMT 18 & 15 & & \\
\hline
\end{tabular}

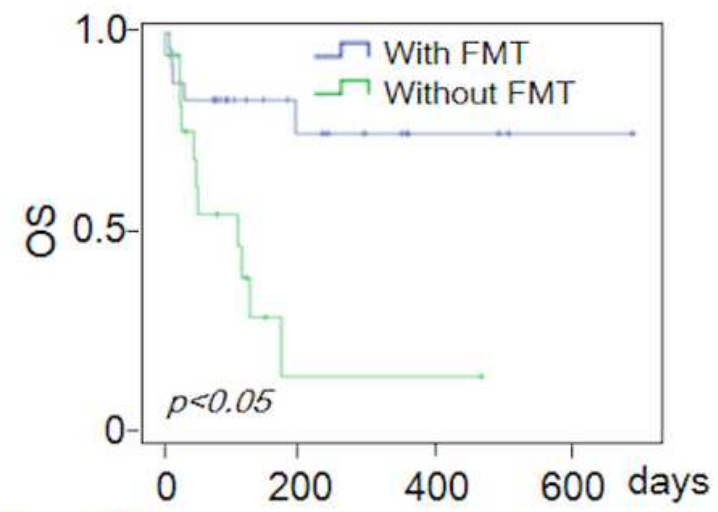

Number at Risk

$\begin{array}{rrrr}\text { With FMT } 24 & 9 & 3 & 1 \\ \text { Without FMT } 18 & 1 & 1 & 0\end{array}$

\section{Figure 3}

Kaplan Meier curves demonstrating survival outcomes EFS (A) and OS (B) of all patients within 90 days of follow up time; EFS (C) and OS (D) at the end of research 
A

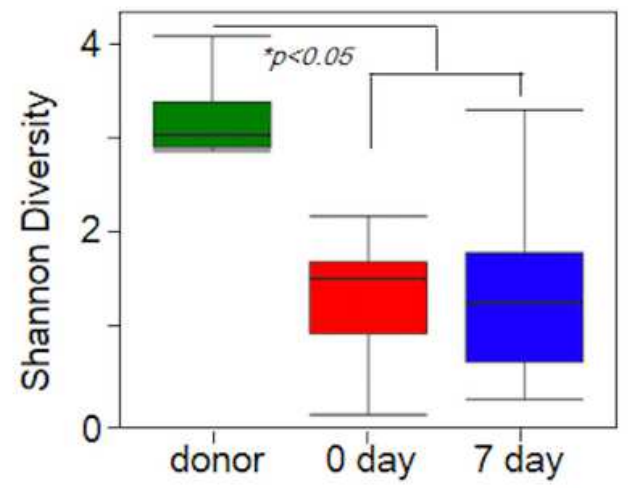

C

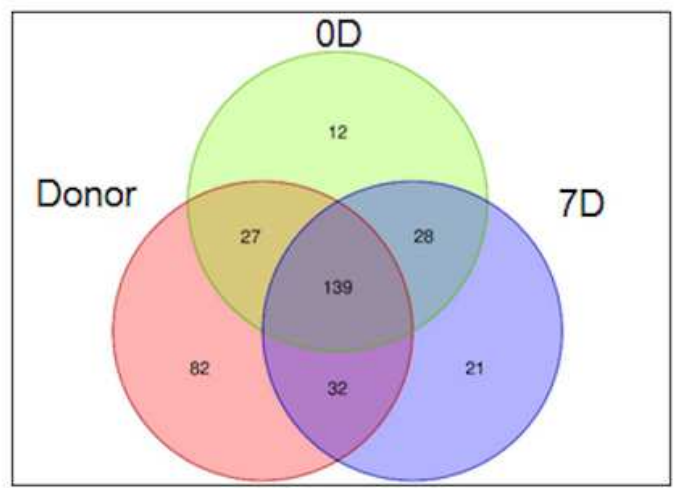

B
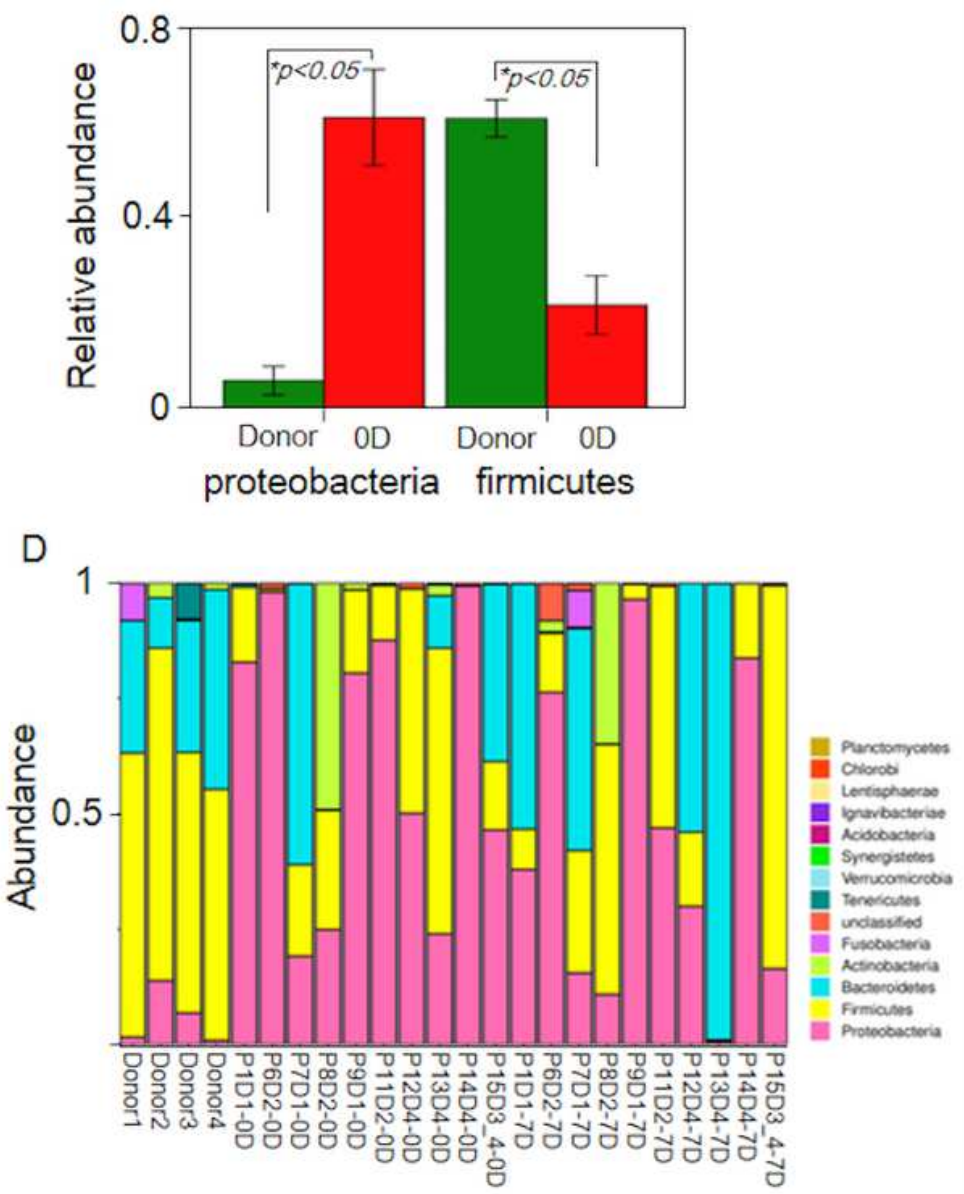

Figure 4

FMT improves gut microbiota diversity and composition in patients. (A) The diversity of fecal microbiota in all sample (Shannon's diversity index) index)(ndonor $=4$, npatient $=10$ ). (B) proteobacteria and firmicutes change in donor group, pre FMT samples. ${ }^{\star} \mathrm{p}<0.05$ (C) OTUs change in donor group, pre FMT (OD) and post FMT (7D) samples. (D) Analysis of fecal microbiota composition in all samples at the phylum level (ndonor $=4$, npatient $=10$ ). Each row represents a study subject. Px means patient number, Dx means donor number, xD means day after FMT.

\section{Supplementary Files}

This is a list of supplementary files associated with this preprint. Click to download.

- Supplementtable1.xlsx

- supplementtable2resultsof28thdays.pdf

- supplementfigureS1.TIF

- supplementmethods.pdf 